\title{
Complex Behavior Analysis of a Fractional-Order Land Dynamical Model with Holling-II Type Land Reclamation Rate on Time Delay
}

\author{
Li Wu, ${ }^{1,2}$ Zhouhong Li $\mathbb{D}^{3}{ }^{3}$ Yuan Zhang, ${ }^{3}$ and Binggeng Xie $\mathbb{D}^{1}$ \\ ${ }^{1}$ College of Resources and Environmental Sciences, Hunan Normal University, Changsha, Hunan 410028, China \\ ${ }^{2}$ Department of Geography and Land Engineering, Yuxi Normal University, Yuxi, Yunnan 653100, China \\ ${ }^{3}$ Department of Mathematics, Yuxi Normal University, Yuxi, Yunnan 653100, China
}

Correspondence should be addressed to Binggeng Xie; xbgyb1961@163.com

Received 14 May 2020; Accepted 25 June 2020; Published 25 July 2020

Guest Editor: Jinliang Wang

Copyright (@) $2020 \mathrm{Li} \mathrm{Wu}$ et al. This is an open access article distributed under the Creative Commons Attribution License, which permits unrestricted use, distribution, and reproduction in any medium, provided the original work is properly cited.

\begin{abstract}
In this paper, a fractional-order land model with Holling-II type transformation rate and time delay is investigated. First of all, the variable-order fractional derivative is defined in the Caputo type. Second, by applying time delay as the bifurcation parameter, some criteria to determine the stability and Hopf bifurcation of the model are presented. It turns out that the time delay can drive the model to be oscillatory, even when its steady state is stable. Finally, one numerical example is proposed to justify the validity of theoretical analysis. These results may provide insights to the development of a reasonable strategy to control land-use change.
\end{abstract}

\section{Introduction}

Since the 20th century, the global environment has changed at an unprecedented speed, and a series of major global environmental problems pose a serious threat to the survival and development of mankind. From the perspective of earth system science, the generation of global environmental problems can be recognized as the result of the interaction between the earth's atmosphere, hydrosphere, biosphere, lithosphere, and human activities $[1,2]$. Land use/land cover change (LUCC) is as a key link connecting the four circles of the earth system, being an ideal entry point for the study of natural and human processes, and has rapidly become the focus of global change research $[3,4]$. LUCC dynamic change is to describe, evaluate, interpret, and predict the quantity, quality, spatial distribution, classification, rate, and process of land use/land cover change by accumulating spatiotemporal continuous land use/land cover data and using the mathematical model [5]. The driving forces of land use change are mainly divided into natural and social systems. Notably, hydrology, topography, and geomorphology $[6,7]$ are the main driving forces in the natural system, while economic development, population growth, and policies are the main driving forces in the social and economic systems [8]. Generally speaking, it is the interwoven factors in these two systems that cause land use changes [9], but compared with the natural factors (such as climate, soil, and topography), human factors (such as regional policy, economic development, and population growth) play a dominant role [10].

Since the key of LUCC research is to understand the driving force and driving mechanism [11], a variety of system analysis and mathematical statistic methods have been well applied in the research of driving force. For example, the internal and human driving force of land use change is studied through the analysis of the multiple linear regression model [12]. The system dynamic model was used to quantitatively diagnose the contribution of each driving factor to land use change [13]. The CLUE comprehensive model was used to establish the interaction between landuse change and its influencing factors, and land change and its spatial distribution were simulated [14-16]. Therefore, the comprehensive mathematical model will be a development trend of LUCC research at present and in the future.

In 1997, Dobson et al. considered the land model with four coupled differential equations [17]: 


$$
\left\{\begin{array}{l}
\dot{F}(t)=-\mathrm{d} P(t) F(t)+s U(t) \\
\dot{A}(t)=\mathrm{d} P(t) F(t)-a A(t)+b U(t) \\
\dot{U}(t)=a A(t)-s U(t)-b U(t) \\
\dot{P}(t)=r P(t)\left[1-\frac{h}{A(t)} P(t)\right]
\end{array}\right.
$$

where $F$ stands for an original area of pristine forest habitat, $A$ denotes the agricultural land, unused land is $U$, and $P$ is the population density with time $t$, for more detail, one can read [17] and the references cited therein. In [18], Chen et al. studied the dynamical analysis of the land model with Holling-II type land reclamation rate, and they found the positive equilibrium point, the basic reproductive rate $R_{0}$, and, furthermore, some sufficient conditions for the global stability of the positive equilibrium and one of the boundary equilibrium.

On the one hand, it is well known that time delays are unavoidable for population modelling. It is therefore important to consider the dynamics for the population models with time delays, and dynamic complex analysis is obviously one of the most important problems. For example, delay differential equations have been applied in the stability of population dynamic systems [19-25], impulsive effects, and control systems [26-28].

On the other hand, there are two types of differential equations, namely, integer-order and fractional-order differential equations. Traditionally, the fractional-order differential equations have enjoyed a preference over integerorder differential equations because of the mathematical tractability of fractional systems. Over the past 30 decades, fractional-order differential equations have been applied in many fields, such as design and control of various ecological systems [29-32], secure communication [33-35], and system control [36-42].

Recently, the existence of Hopf bifurcation of differential equations has been studied as an important qualitative behavior of integer-order differential equations [43]. Moreover, fractional calculus is merged into complicated, dynamical systems which extremely renovate the theory of the design and control performance for complex systems. It has been discovered that physical phenomena in nature can be depicted more accurately by fractional-order models in comparison with classical integer-order ones [44, 45]. Some scholars introduced fractional calculus into population models and constructed fractional population or epidemic models [46-50], fractional dynamic systems, and fractional neural networks [51-53]. However, to the best of our knowledge, there are few studies to investigate the existence of Hopf bifurcation to the fractional-order land model with time delay.

Motived by the above ideas, in this paper, we will consider the following fractional-order land model with Holling-II type response and time delay:

$$
\left\{\begin{array}{l}
D^{\phi} F(t)=\frac{-\mathrm{d} F(t) P(t-\tau)}{1+P(t-\tau)}+s U(t), \\
D^{\phi} R(t)=\frac{\mathrm{d} F(t) P(t-\tau)}{1+P(t-\tau)}-a R(t)+b U(t), \\
D^{\phi} U(t)=a R(t)-s U(t)-b U(t), \\
D^{\phi} P(t)=r P(t)\left[1-\frac{h}{R(t)} P(t-\tau)\right],
\end{array}\right.
$$

where $\phi \in(0,1]$ is the fractional order, $F(t), R(t)$, and $U(t)$ represent the land of wood and grass, survival land, and unused land, respectively, and $P(t)$ is the density of the predator population at time $t ; R(t)$ (land area of survival) of a period of time $1 / a$ becomes unused land (area $U(t)$ ), which in turn recovers through natural succession or ecological restoration to become a forest after a time interval $1 / s$. The unused land may also be restored to survival viable land after a time interval $1 / b$. Average clearing ability is described by constant $d$. $r$ is the growth rate into the population. $\tau>0$ is the time delay required for the gestation of the mature population.

The main contributions can be summed up in three aspects:

(1) A novel fractional-order land model with Holling-II type land reclamation rate and time delay is formulated

(2) Two primary dynamical properties-stability and oscillation-of the delayed fractional-order land model are investigated

(3) The influences of the order on the Hopf bifurcation are obtained

Throughout this paper, we address the following assumptions.

Assumption 1. bd+ds-ahs $>0$.

Assumption 2. $a b d+a d s-h a^{2} s>0$.

Suppose Assumptions 1 and 2 hold, then system (2) has an unique positive equilibrium point $E^{*}=\left(F^{*}, R^{*}, U^{*}, P^{*}\right)$, which is described by

$$
\begin{aligned}
F^{*} & =\frac{a s(a h+b h+b+h s+s)}{(b+s)(a d+a s+b d+d s)}, \\
R^{*} & =\frac{b d+d s-a h s}{a d+a s+b d+d s}, \\
U^{*} & =\frac{a b d+a d s-h a^{2} s}{(b+s)(a d+a s+b d+d s)}, \\
P^{*} & =\frac{b d+d s-a h s}{h(a d+a s+b d+d s)} .
\end{aligned}
$$

The remainder of the current paper is organized as follows: In Section 2, some definitions and lemmas of fractional calculus are recalled. In Section 3, the fractional 
land model with Holling-II type land reclamation rate and time delay is investigated by using time delay as the bifurcation parameter, and the conditions of Hopf bifurcation are presented. One numerical example is given to illustrate the effectiveness of our main results in Section 4. Finally, conclusions are drawn in the last section.

\section{Basic Tools of Fractional Calculus}

In this section, we recall some definitions and lemmas of fractional calculus, which can be used in the proofs of the main results in Section 3.

There are several definitions of fractional derivatives. The Grünwald-Letnikov definition, the Riemann-Liouville definition, and the Caputo definition are usually used to deal with fractional-order systems. Since the Caputo derivative only requires the initial conditions which are based on the integer-order derivative and represents well-understood features of physical situation, it is more applicable to realworld problems. Hence, the Caputo fractional-order derivative is adopted in this paper.

Definition 1 (see [44]). The fractional integral of order $\alpha$ for a function $g(t)$ is defined as

$$
I^{\alpha} g(t)=\frac{1}{\Gamma(\alpha)} \int_{t_{0}}^{t}(t-s)^{\alpha-1} g(s) \mathrm{d} s,
$$

where $t \geq t_{0}, \alpha>0, \Gamma(\cdot)$ is the gamma function, and $\Gamma(s)=\int_{0}^{\infty} t^{s-1} e^{-t} \mathrm{~d} t$.

Definition 2 (see [44]). The Caputo fractional derivative of order $\alpha$ for a function $g(t) \in C^{n}\left(\left[t_{0}, \infty\right), R\right)$ is defined by

$$
D^{\alpha} g(t)=\frac{1}{\Gamma(n-\alpha)} \int_{t_{0}}^{t} \frac{g^{(n)}(s)}{(t-s)^{\alpha-n+1}} \mathrm{~d} s,
$$

where $t \geq t_{0}$ and $n$ is a positive integer such that $n-1 \leq \alpha<n$.

Moreover, when $0<\alpha<1$,

$$
D^{\alpha} g(t)=\frac{1}{\Gamma(1-\alpha)} \int_{t_{0}}^{t} \frac{g^{\prime}(s)}{(t-s)^{\alpha}} \mathrm{d} s .
$$

Lemma 1 (see [41]). The following autonomous system

$$
\begin{aligned}
& D^{\alpha} y=J y, \\
& y(0)=y_{0},
\end{aligned}
$$

where $0<\alpha<1, y \in R^{n}$, and $A \in R^{n \times n}$, is asymptotically stable if and only if $\left|\arg \left(\lambda_{i}\right)\right|>\alpha \pi / 2(i=1,2, \ldots, n)$. In this case, each component of the states decays towards 0 like $t^{-\alpha}$. Also, this system is stable if and only if $\left|\arg \left(\lambda_{i}\right)\right| \geq \alpha \pi / 2$, and those critical eigenvalues that satisfy $\left|\arg \left(\lambda_{i}\right)\right|=\alpha \pi / 2$ have geometric multiplicity one.

\section{Main Results}

In this section, by applying the previous analytic technique, we shall investigate the stability and bifurcation of system (2) with time delay by taking time delay as the bifurcation parameter. The conditions of delay-induced bifurcation will be derived.

From reference [18], the dynamics of system (2) crucially depend on the basic reproduction number $R_{0}$, which is given by

$$
R_{0}=\frac{b d+d s}{a s h}
$$

Let $x_{1}(t)=F(t)-F^{*}, x_{2}(t)=R(t)-R^{*}, x_{3}(t)=U(t)-$ $U^{*}$, and $x_{4}(t)=P(t)-P^{*}$, and then we have

$$
\left\{\begin{array}{l}
D^{\phi} x_{1}(t)=\frac{-d\left(x_{1}(t)+F^{*}\right)\left(x_{4}(t-\tau)+P^{*}\right)}{1+\left(x_{4}(t-\tau)+P^{*}\right)}+s\left(x_{3}(t)+U^{*}\right), \\
D^{\phi} x_{2}(t)=\frac{d\left(x_{1}(t)+F^{*}\right)\left(x_{4}(t-\tau)+P^{*}\right)}{1+\left(x_{4}(t-\tau)+P^{*}\right)}-a\left(x_{2}(t)+R^{*}\right)+b\left(x_{3}(t)+U^{*}\right), \\
D^{\phi} x_{3}(t)=a\left(x_{2}(t)+R^{*}\right)-s\left(x_{3}(t)+U^{*}\right)-b\left(x_{3}(t)+U^{*}\right), \\
D^{\phi} x_{4}(t)=r\left(x_{4}(t)+P^{*}\right)\left[1-\frac{h}{\left(x_{2}(t)+R^{*}\right)}\left(x_{4}(t-\tau)+P^{*}\right)\right]
\end{array}\right.
$$

Taking advantage of the Taylor expansion formula, the linearized system of system (9) at the zero equilibrium is

$$
\left\{\begin{array}{l}
D^{\phi} x_{1}(t)=a_{11} x_{1}(t)+a_{13} x_{3}(t)+b_{14} x_{4}(t-\tau), \\
D^{\phi} x_{2}(t)=a_{21} x_{1}(t)+a_{22} x_{2}(t)+a_{23} x_{3}(t)+b_{24} x_{4}(t-\tau), \\
D^{\phi} x_{3}(t)=a_{32} x_{2}(t)+a_{33} x_{3}(t), \\
D^{\phi} x_{4}(t)=a_{42} x_{2}(t)+b_{44} x_{4}(t-\tau),
\end{array}\right.
$$


where $a_{11}=\left(-d P^{*} /\left(1+P^{*}\right)\right), a_{13}=s, b_{14}=-\left(d F^{*} /((1+\right.$ $\left.\left.\left.P^{*}\right)^{2}\right)\right), a_{21}=\left(d P^{*} /\left(1+P^{*}\right)\right), a_{22}=-a, a_{23}=b, b_{24}=\left(d F^{*} /\right.$ $\left.\left(\left(1+P^{*}\right)^{2}\right)\right), a_{32}=a, a_{33}=-(b+s), a_{42}=\left(h r P^{* 2} / R^{* 2}\right), a_{44}=$ $r-\left(h r P^{*} / R^{*}\right)$, and $b_{44}=-\left(h r P^{*} / R^{*}\right)$.

The associated characteristic equation of system (10) can be obtained as

$$
\operatorname{det}\left(\begin{array}{cccc}
s^{\phi}-a_{11} & 0 & -a_{13} & -b_{14} e^{-s \tau} \\
-a_{21} & s^{\phi}-a_{22} & -a_{23} & -b_{24} e^{-s \tau} \\
0 & -a_{32} & s^{\phi}-a_{33} & 0 \\
0 & -a_{42} & 0 & s^{\phi}-a_{44}-b_{44} e^{-s \tau}
\end{array}\right)=0
$$

which leads to

$$
P_{1}(s)+P_{2}(s) e^{-s \tau}=0
$$

where

$$
\begin{aligned}
P_{1}(s)= & \left(-a_{11} s^{2 \phi}-a_{22} s^{2 \phi}-a_{33} s^{2 \phi}+a_{11} a_{22} s^{\phi}\right. \\
& -a_{23} a_{32} s^{\phi}+a_{11} a_{33} s^{\phi}+a_{22} a_{33} s^{\phi} \\
& \left.-a_{12} a_{21} a_{32}+a_{11} a_{23} a_{32}-a_{11} a_{22} a_{33}+s^{3 \phi}\right)\left(s^{\phi}-a_{44}\right), \\
P_{2}(s)= & a_{21}\left(a_{12} a_{32} b_{44}-a_{42} b_{14}\left(s^{\phi}-a_{33}\right)\right) \\
& -\left(s^{\phi}-a_{11}\right)\left(a_{42} b_{24}\left(s^{\phi}-a_{33}\right)\right.
\end{aligned}
$$

$$
\left.+b_{44}\left(-a_{33} s^{\phi}+a_{22}\left(a_{33}-s^{\phi}\right)-a_{23} a_{32}+s^{2 \phi}\right)\right) .
$$

Let $P_{1}(s)=A_{1}+i B_{1}$ and $P_{2}(s)=A_{2}+i B_{2}$, and from equation (12), we have

$$
\left(A_{1}+i B_{1}\right)+\left(A_{2}+i B_{2}\right) e^{-s \tau}=0 .
$$

Assume that $s=i w=w(\cos (\pi / 2)+i \sin (\pi / 2))$ is a root of equation (12), $\omega>0$. Substituting $s$ into equation (12) and separating the real and imaginary parts, one can have

$$
\left\{\begin{array}{l}
A_{2} \cos w \tau+B_{2} \sin w \tau=-A_{1}, \\
B_{2} \cos w \tau-A_{2} \sin w \tau=-B_{1} .
\end{array}\right.
$$

Applying equation (15), direct calculation yields

$$
\left\{\begin{array}{l}
\cos w \tau=-\frac{A_{1} A_{2}+B_{1} B_{2}}{A_{2}^{2}+B_{2}^{2}}=\mathscr{F}(w), \\
\sin w \tau=-\frac{-A_{2} B_{1}+A_{1} B_{2}}{A_{2}^{2}+B_{2}^{2}}=\mathscr{G}(w),
\end{array}\right.
$$

where

$$
\begin{aligned}
A_{1}= & \left(-a_{23} a_{32}+a_{22} a_{33}+\left(a_{22}+a_{33}\right) a_{44}+a_{11}\left(a_{22}+a_{33}+a_{44}\right)\right) w^{2 \phi} \cos (\pi \phi) \\
& -\left(a_{11}+a_{22}+a_{33}+a_{44}\right) w^{3 \phi} \cos \left(\frac{3 \pi \phi}{2}\right)+\left(a_{12} a_{21} a_{32}+\left(a_{22} a_{33}-a_{23} a_{32}\right) a_{44}\right. \\
& \left.+a_{11}\left(-a_{23} a_{32}+a_{22} a_{33}+\left(a_{22}+a_{33}\right) a_{44}\right)\right) w^{\phi}\left(-\cos \left(\frac{\pi \phi}{2}\right)\right)+\left(a_{12} a_{21} a_{32}\right. \\
& \left.+a_{11}\left(a_{22} a_{33}-a_{23} a_{32}\right)\right) a_{44}+w^{4 \phi} \cos (2 \pi \phi), \\
B_{1}=a_{21} & \left(a_{12} a_{32} b_{44}-a_{42} b_{14}\left(w^{\phi} \cos \left(\frac{\pi \phi}{2}\right)-a_{33}\right)\right)+a_{42} b_{24}\left(-\left(\left(a_{11}+a_{33}\right) w^{\phi}\left(-\cos \left(\frac{\pi \phi}{2}\right)\right)\right.\right. \\
& \left.\left.+a_{11} a_{33}+w^{2 \phi} \cos (\pi \phi)\right)\right)+b_{44}\left(\left(a_{11}+a_{22}+a_{33}\right) w^{2 \phi} \cos (\pi \phi)+\left(a_{11} a_{22}-a_{23} a_{32}\right.\right. \\
& \left.\left.+\left(a_{11}+a_{22}\right) a_{33}\right) w^{\phi}\left(-\cos \left(\frac{\pi \phi}{2}\right)\right)+a_{11}\left(a_{22} a_{33}-a_{23} a_{32}\right)-w^{3 \phi} \cos \left(\frac{3 \pi \phi}{2}\right)\right), \\
& \left.+a_{21} a_{42} b_{14}\right) . \\
A_{2}= & a_{21}\left(a_{12} a_{32} b_{44}-a_{42} b_{14}\left(w^{\phi} \cos \left(\frac{\pi \phi}{2}\right)-a_{33}\right)\right)+a_{42} b_{24}\left(-\left(\left(a_{11}+a_{33}\right) w^{\phi}\left(-\cos \left(\frac{\pi \phi}{2}\right)\right)\right.\right. \\
& \left.\left.+a_{11} a_{33}+w^{2 \phi} \cos (\pi \phi)\right)\right)+b_{44}\left(\left(a_{11}+a_{22}+a_{33}\right) w^{2 \phi} \cos (\pi \phi)+\left(a_{11} a_{22}-a_{23} a_{32}\right.\right. \\
& \left.\left.+\left(a_{11}+a_{22}\right) a_{33}\right) w^{\phi}\left(-\cos \left(\frac{\pi \phi}{2}\right)\right)+a_{11}\left(a_{22} a_{33}-a_{23} a_{32}\right)-w^{3 \phi} \cos \left(\frac{3 \pi \phi}{2}\right)\right), \\
B_{2}= & -w^{\phi} \sin \left(\frac{\pi \phi}{2}\right)\left(a_{42} b_{24}\left(-a_{11}-a_{33}+2 w^{\phi} \cos \left(\frac{\pi \phi}{2}\right)\right)+b_{44}\left(-2\left(a_{11}+a_{22}+a_{33}\right)\right.\right. \\
& \left.+a_{23} a_{32}+a_{11} a_{33}+a_{22} a_{33}+w^{2 \phi}(2 \cos (\pi \phi)+1)\right) \\
& \left.+\frac{\pi}{2}\right)
\end{aligned}
$$


From (16), we obtain

$$
\tau^{(k)}=\frac{1}{w}[\arccos \mathscr{F}(w)+2 k \pi], \quad k=0,1,2, \ldots
$$

Define

$$
\tau_{0}^{*}=\min \left\{\tau^{(k)}\right\}, \quad k=0,1,2, \ldots,
$$

where $\tau^{(k)}$ is defined by equation (18).

To derive the condition of the occurrence for Hopf bifurcation, we further give the following assumption.

Assumption 3

$$
\frac{W_{1} V_{1}+W_{2} V_{2}}{V_{1}^{2}+V_{2}^{2}} \neq 0
$$

where

$$
\begin{aligned}
& W_{1}=w_{0}\left(A_{2} \sin w_{0} \tau_{0}-B_{2} \cos w_{0} \tau_{0}\right) \\
& W_{2}=w_{0}\left(A_{2} \cos w_{0} \tau_{0}+B_{2} \sin w_{0} \tau_{0}\right) \\
& V_{1}=A_{1}^{\prime}+\left(A_{2}^{\prime}-\tau_{0} A_{2}\right) \cos w_{0} \tau_{0}+\left(B_{2}^{\prime}-\tau_{0} B_{2}\right) \sin w_{0} \tau_{0} \\
& V_{2}=B_{1}^{\prime}+\left(B_{2}^{\prime}-\tau_{0} B_{2}\right) \cos w_{0} \tau_{0}\left(A_{2}^{\prime}-\tau_{0} A_{2}\right) \sin w_{0} \tau_{0} .
\end{aligned}
$$

Lemma 2. Let $s(\tau)=\nu(\tau)+i w(\tau)$ be the root of equation (18) near $\tau=\tau_{j}$ satisfying $\nu\left(\tau_{j}\right)=0, w\left(\tau_{j}\right)=w_{0}$, then the following transversality condition holds

$$
\left.\operatorname{Re}\left[\frac{\mathrm{d} s}{\mathrm{~d} \tau}\right]\right|_{\left(w=w_{0}, \tau=\tau_{0}\right)} \neq 0
$$

Proof. By using implicit function theorem and differentiating (12) with respect to $\tau$, we have

$$
P_{1}^{\prime}(s) \frac{\mathrm{d} s}{\mathrm{~d} \tau}+P_{2}^{\prime}(s) e^{-s \tau} \frac{\mathrm{d} s}{\mathrm{~d} \tau}+P_{2}(s) e^{-s \tau}\left(-\tau \frac{\mathrm{d} s}{\mathrm{~d} \tau}-s\right)=0
$$

where $P_{i}^{\prime}(s)$ are the derivatives of $P_{i}(s)(i=1,2)$.

Thus, we obtain

$$
\frac{\mathrm{d} s}{\mathrm{~d} \tau}=\frac{W(s)}{V(s)}
$$

where

$$
\begin{aligned}
& W(s)=s P_{2}(s) e^{-s \tau}, \\
& V(s)=P_{1}^{\prime}(s)+P_{2}^{\prime}(s) e^{-s \tau}-\tau P_{2}(s) e^{-s \tau} .
\end{aligned}
$$

Let $P_{i}^{R}$ and $P_{i}^{I}$ be the real and imaginary parts of $P_{i}(s)(i=1,2)$, respectively. We further suppose that $W_{1}$ and $W_{2}$ are the real and imaginary parts of $W(s)$, respectively, and $V_{1}, V_{2}$ are the real and imaginary parts of $V(s)$, respectively.

Hence,

$$
\left.\operatorname{Re}\left[\frac{\mathrm{d} s}{\mathrm{~d} \tau}\right]\right|_{\left(\tau=\tau_{0}, w=w_{0}\right)}=\frac{W_{1} V_{1}+W_{2} V_{2}}{V_{1}^{2}+V_{2}^{2}}
$$

Therefore, based on Assumption 3, the transversality condition is satisfied. This completes the proof of Lemma 2.

Denote

$$
\begin{aligned}
& \beta_{1}=\alpha_{1}, \\
& \beta_{2}=\left|\begin{array}{ll}
\alpha_{1} & 1 \\
\alpha_{3} & \alpha_{2}
\end{array}\right|, \\
& \beta_{3}=\left|\begin{array}{lll}
\alpha_{1} & 1 & 0 \\
\alpha_{3} & \alpha_{2} & \alpha_{1} \\
0 & \alpha_{4} & \alpha_{3}
\end{array}\right|, \\
& \beta_{4}=\alpha_{3} \beta_{3},
\end{aligned}
$$

where

$$
\begin{aligned}
\alpha_{1}= & -a_{11}-a_{22}-a_{33}-a_{44}-b_{44}, \\
\alpha_{2}= & a_{22} b_{44}-a_{42} b_{24}+a_{11} b_{44}+a_{33} b_{44}+a_{11} a_{22}+a_{33} a_{22} \\
& +a_{44} a_{22}-a_{23} a_{32}+a_{11} a_{33}+a_{11} a_{44}+a_{33} a_{44}, \\
\alpha_{3}= & a_{23} a_{32} b_{44}-a_{21} a_{42} b_{14}+a_{11} a_{42} b_{24}+a_{33} a_{42} b_{24} \\
& -a_{11} a_{22} b_{44}-a_{11} a_{33} b_{44}-a_{22} a_{33} b_{44} \\
& -a_{13} a_{21} a_{32}+a_{11} a_{23} a_{32}+a_{23} a_{44} a_{32}-a_{11} a_{22} a_{33} \\
& -a_{11} a_{22} a_{44}-a_{11} a_{33} a_{44}-a_{22} a_{33} a_{44}, \\
\alpha_{4}= & a_{21} a_{33} a_{42} b_{14}-a_{11} a_{33} a_{42} b_{24} \\
& +a_{13} a_{21} a_{32} a_{44}-a_{11} a_{23} a_{32} a_{44}+a_{11} a_{22} a_{33} a_{44} \\
& +a_{13} a_{21} a_{32} b_{44}-a_{11} a_{23} a_{32} b_{44}+a_{11} a_{22} a_{33} b_{44} .
\end{aligned}
$$

Lemma 3. If Assumptions 1 and 2 hold, then the positive equilibrium point $\left(F^{*}, R^{*}, U^{*}, P^{*}\right)$ of the delayed fractionalorder land model (2) is asymptotically stable when $\tau=0$.

Proof. When $\tau=0$, system (2) changes into

$$
\lambda^{4}+\beta_{1} \lambda^{3}+\beta_{2} \lambda^{2}+\beta_{3} \lambda+\beta_{4}=0 \text {. }
$$

If the conditions of $\beta_{i}>0(i=1,2,3,4)$ hold and, furthermore, if Assumptions 1 and 2 are satisfied, it is easy to check from the Routh-Hurwitz criterion that the four eigenvalues of the characteristic equation (29) have negative real parts. Hence, the positive equilibrium point $\left(F^{*}, R^{*}, U^{*}, P^{*}\right)$ of the delayed fractional-order land model (2) is asymptotically stable when $\tau=0$.

From the above analysis, together with Lemma 3, we will give the following theorem.

Theorem 1. For the model (2):

(i) Under Assumptions 1-3, the equilibrium point $E^{*}=$ $\left(F^{*}, R^{*}, U^{*}, P^{*}\right)$ is global asymptotically stable for $\tau \in[0,+\infty)$

(ii) Under Assumptions 1-3:

(a) The equilibrium point $E^{*}=\left(F^{*}, R^{*}, U^{*}, P^{*}\right)$ is locally asymptotically stable for $\tau \in\left[0, \tau_{0}\right)$ 


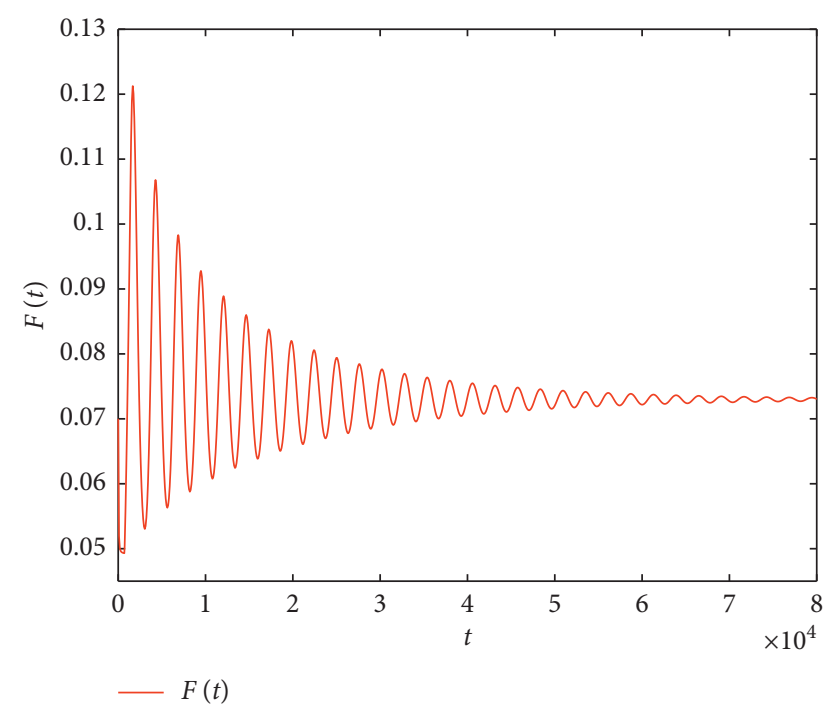

Figure 1: Time series solution of system (30) with $\phi=0.90$, $\tau=700<\tau_{0}=728.403$.

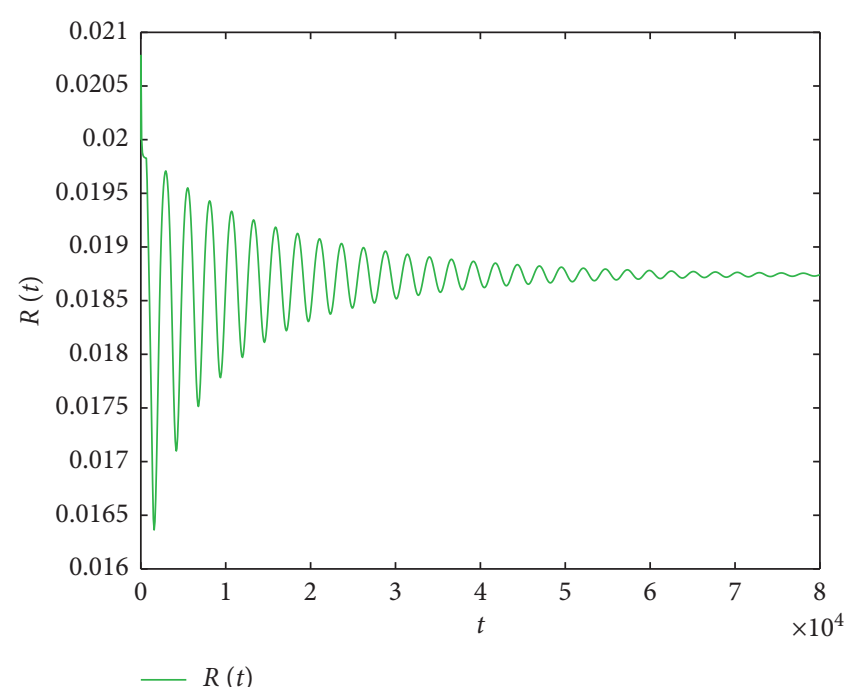

Figure 2: Time series solution of system (30) with $\phi=0.90$, $\tau=700<\tau_{0}=728.403$.

(b) Model (2) undergoes a Hopf bifurcation at the $E^{*}=\left(F^{*}, R^{*}, U^{*}, P^{*}\right)$ when $\tau=\tau_{0}$, i.e., it has a branch of periodic solutions bifurcating from the equilibrium near $\tau=\tau_{0}$

\section{Numerical Example and Application}

In this section, we give one example to demonstrate the effectiveness of the proposed approach. The simulation results are based on theAdams-Bashforth-Moulton predictor-corrector scheme [54], and step length $\Delta t=0.01$.

Consider the following fractional-order land dynamical model with time delay:

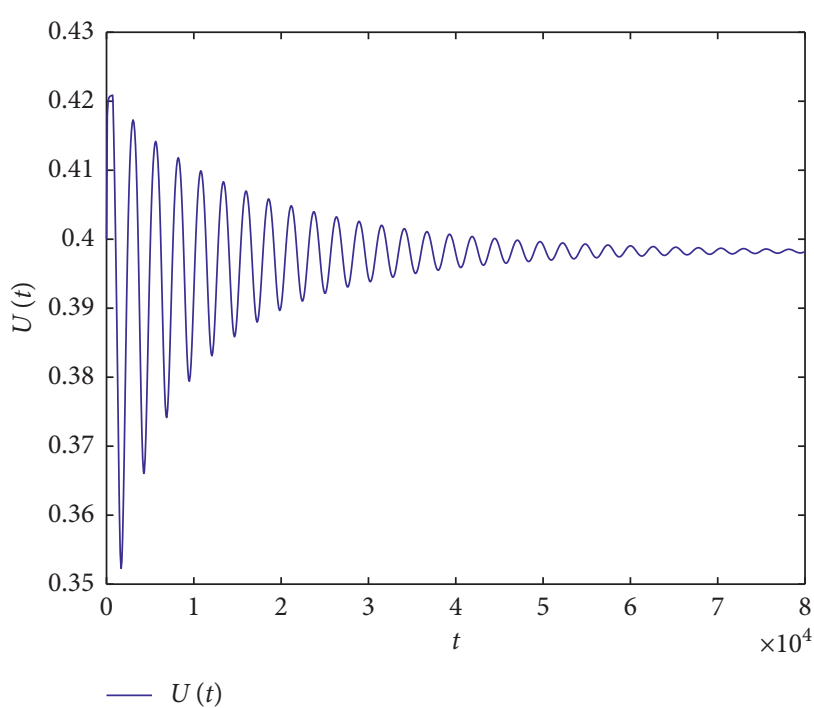

FIgURE 3: Time series solution of system (30) with $\phi=0.90$, $\tau=700<\tau_{0}=728.403$.

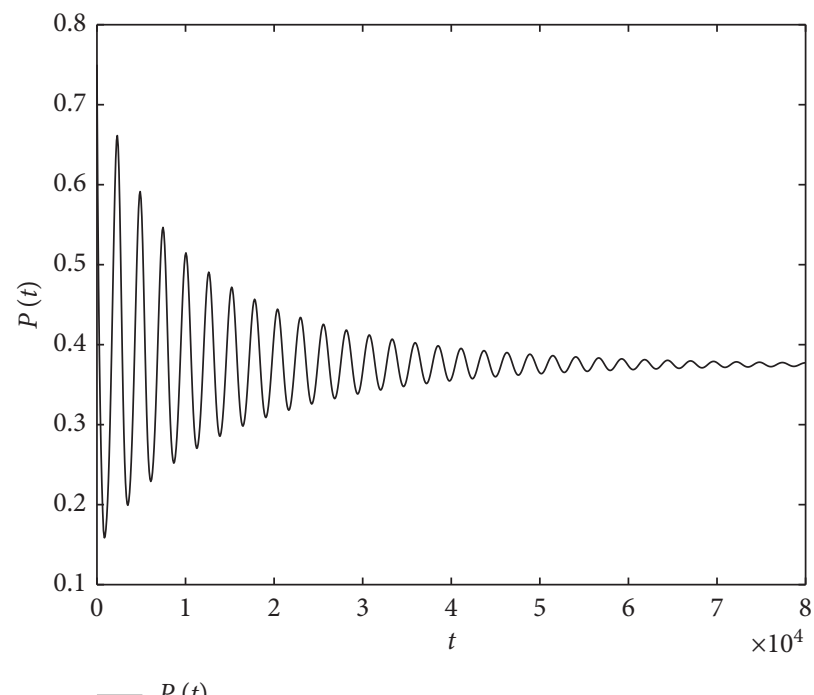

FIgURE 4: Time series solution of system (30) with $\phi=0.90$, $\tau=700<\tau_{0}=728.403$.

$$
\left\{\begin{array}{l}
D^{\phi} F(t)=\frac{-\mathrm{d} F(t) P(t-\tau)}{1+P(t-\tau)}+s U(t), \\
D^{\phi} R(t)=\frac{\mathrm{d} F(t) P(t-\tau)}{1+P(t-\tau)}-a R(t)+b U(t), \\
D^{\phi} U(t)=a R(t)-s U(t)-b U(t), \\
D^{\phi} P(t)=r P(t)\left[1-\frac{h}{R(t)} P(t-\tau)\right],
\end{array}\right.
$$




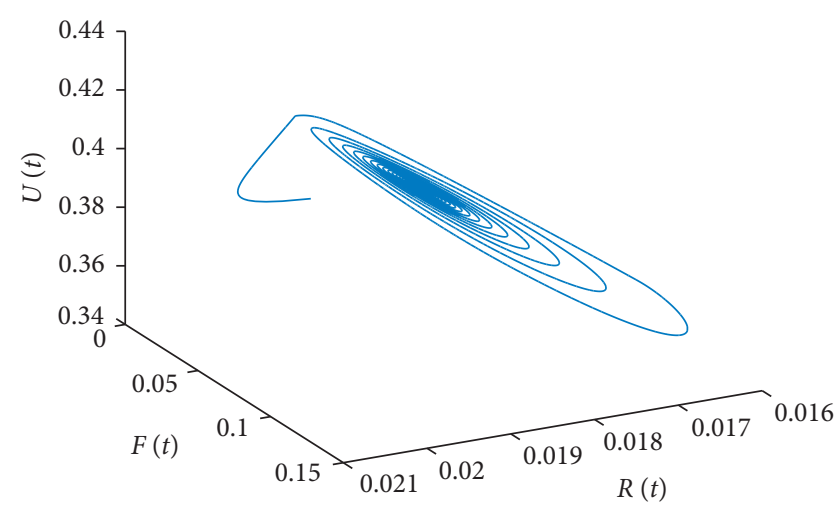

Figure 5: Portrait diagram of land model (30) with $\phi=0.90$, $\tau=700<\tau_{0}=728.403$.

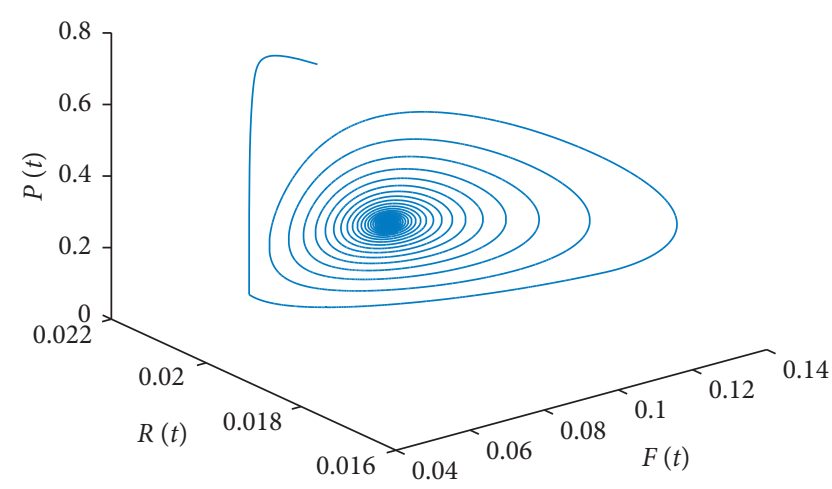

FIgURE 6: Portrait diagram of land model (30) with $\phi=0.90$, $\tau=700<\tau_{0}=728.403$.

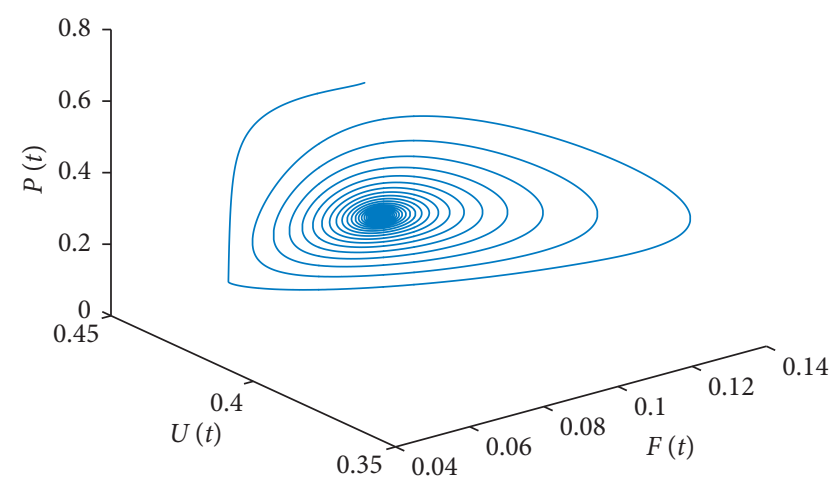

Figure 7: Portrait diagram of land model (30) with $\phi=0.90$, $\tau=700<\tau_{0}=728.403$.

in this case, taking $\phi=0.9$, and according to Yuxi city land and population data of Yunan Province, China, let $a=0.34, b=0.012, r=0.0048, s=0.004, h=0.05$, and $d=$ 0.08 . By computing, we obtain the positive equilibrium point as $(0.075617,0.021169,0.44984,0.423379)$. Moreover, we have $R_{0}=((b d+d s) / a s h)=18.8235>1, \quad \omega_{0}=0.002358$, and $\tau_{0}=728.403$. Furthermore, the initial values are selected

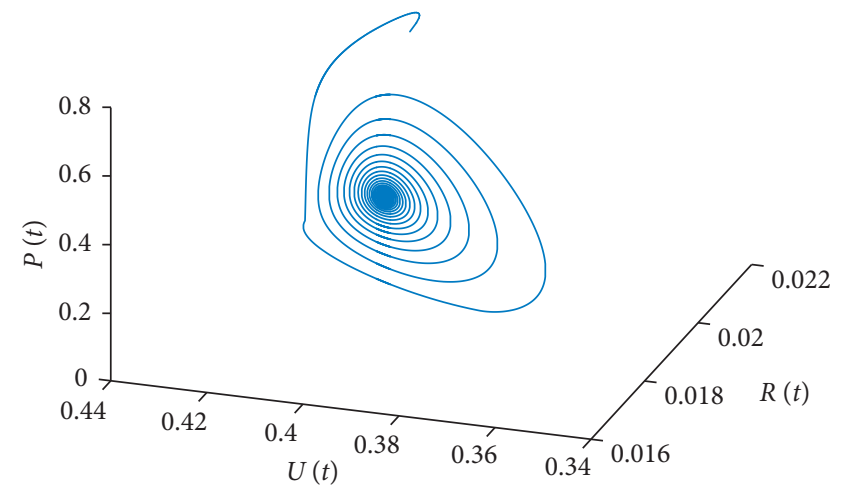

Figure 8: Portrait diagram of land model (30) with $\phi=0.90$, $\tau=700<\tau_{0}=728.403$.

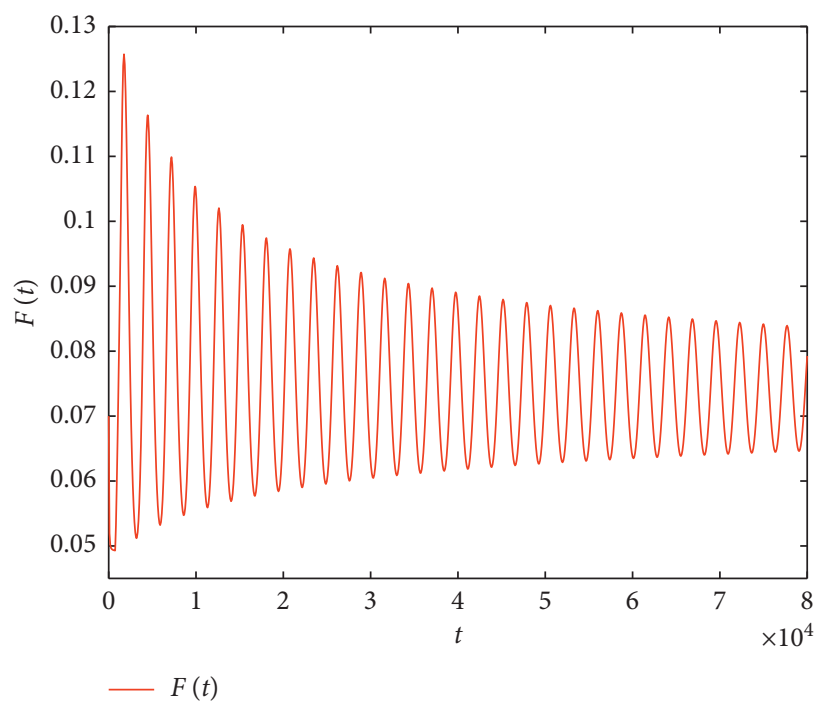

FIgURE 9: Time series solution of system (30) with $\phi=0.90$, $\tau=740>\tau_{0}=728.403$.

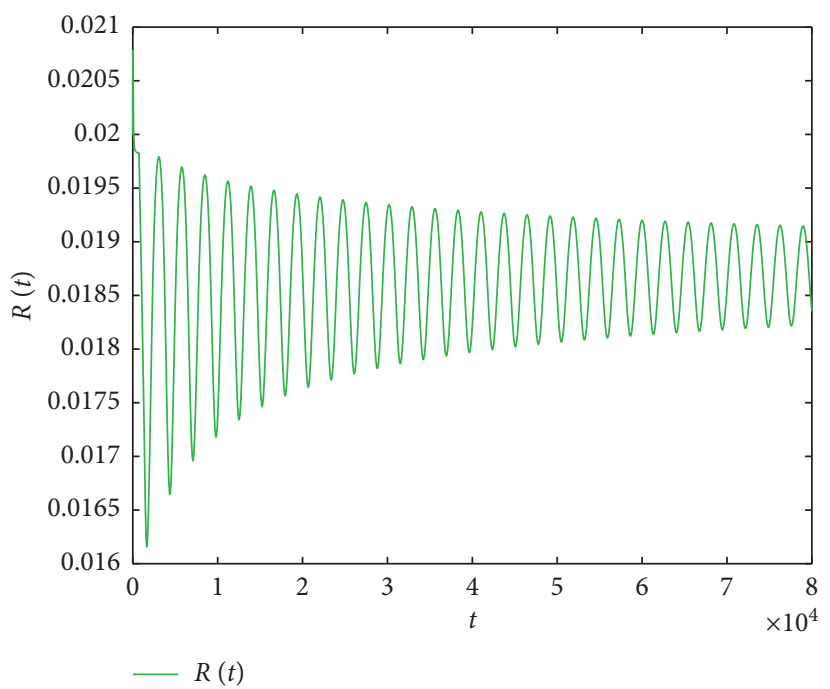

FIgUre 10: Time series solution of system (30) with $\phi=0.90$, $\tau=740>\tau_{0}=728.403$. 


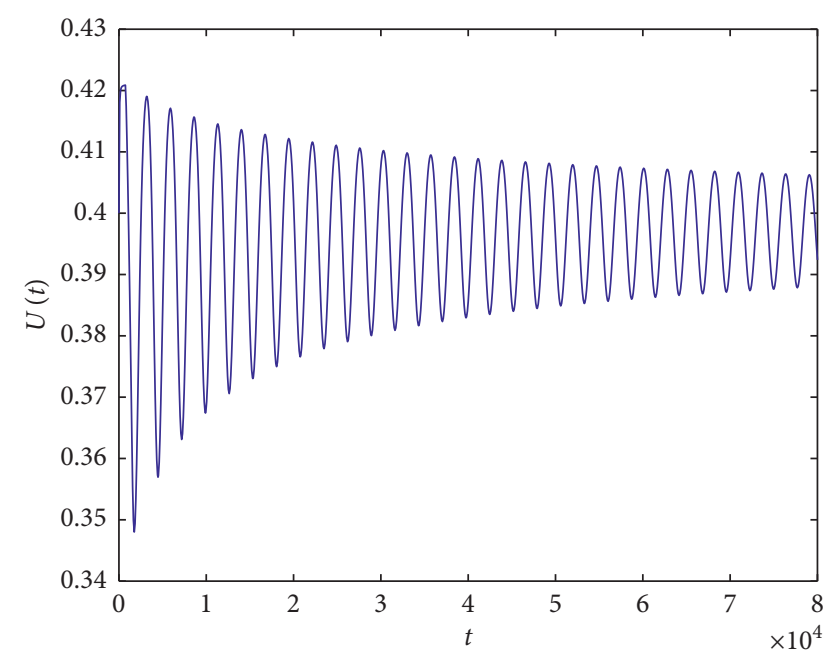

$-U(t)$

FIgUre 11: Time series solution of system (30) with $\phi=0.90$, $\tau=740>\tau_{0}=728.403$.

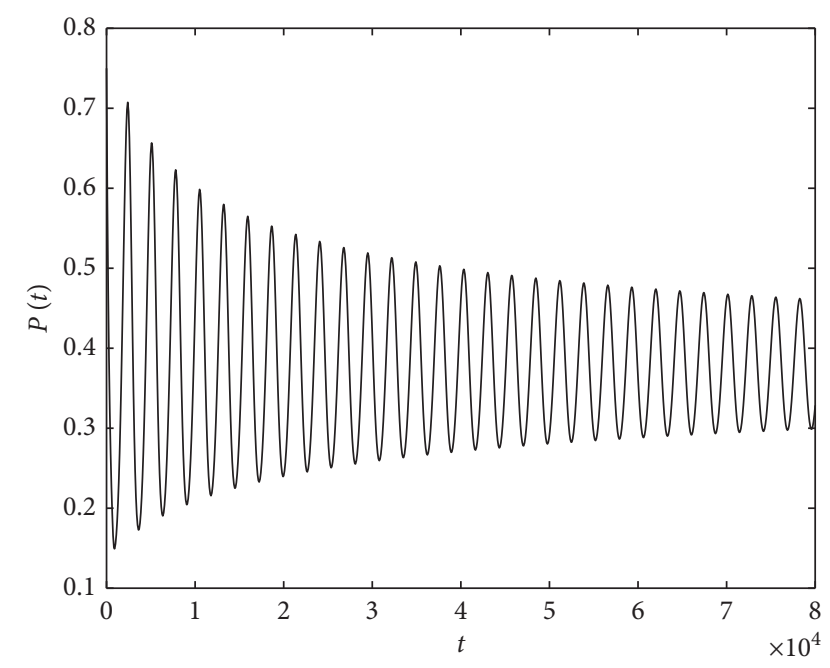

$-P(t)$

Figure 12: Time series solution of system (30) with $\phi=0.90$, $\tau=740>\tau_{0}=728.403$.

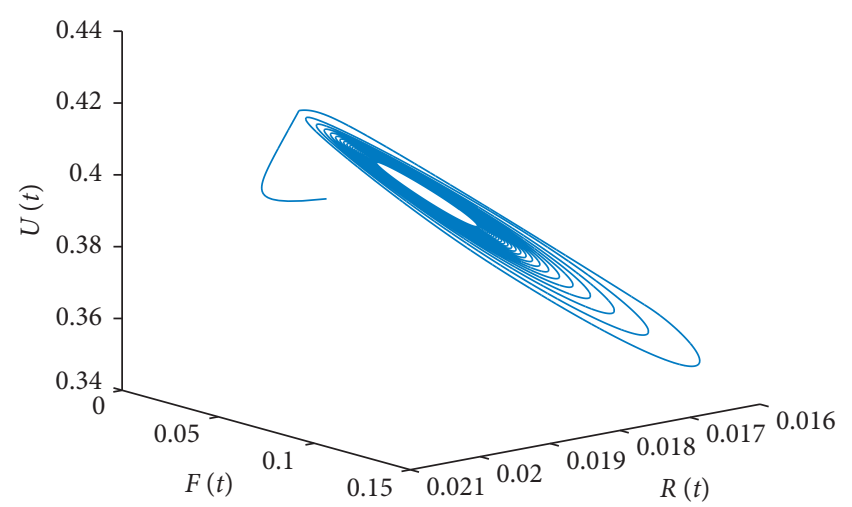

FIgURE 13: Portrait diagram of land model (30) with $\phi=0.90$, $\tau=740>\tau_{0}=728.403$.

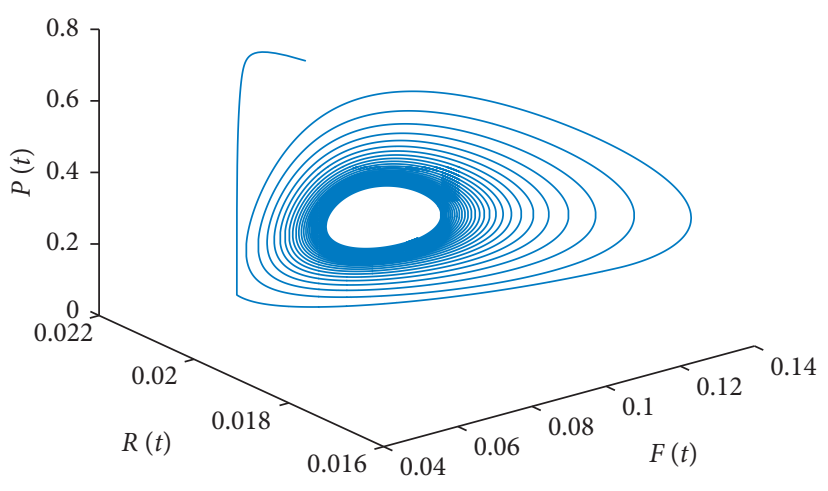

FIgURE 14: Portrait diagram of land model (30) with $\phi=0.90$, $\tau=740>\tau_{0}=728.403$.

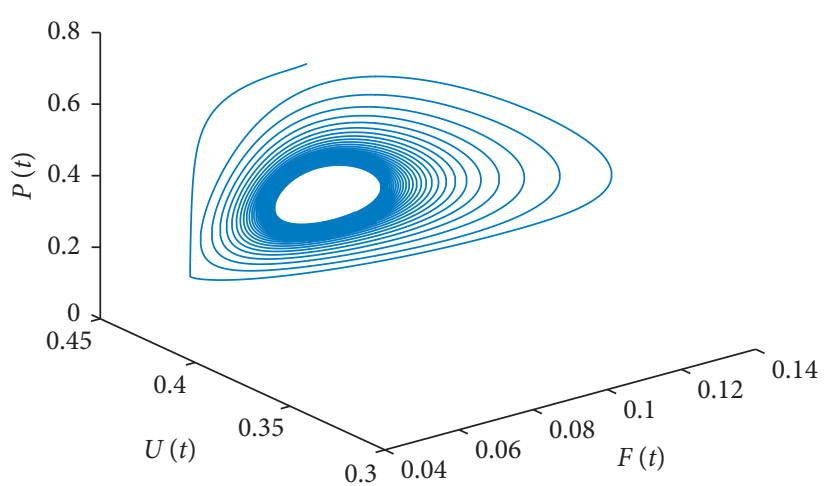

FIgURE 15: Portrait diagram of land model (30) with $\phi=0.90$, $\tau=740>\tau_{0}=728.403$.

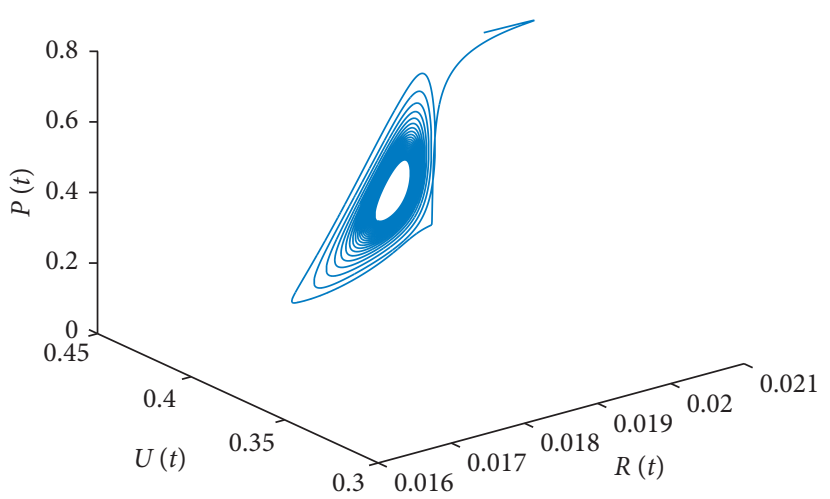

FIgURE 16: Portrait diagram of land model (30) with $\phi=0.90$, $\tau=740>\tau_{0}=728.403$.

as $(F(0), R(0), U(0), P(0))=(0.07,0.02,0.4,0.75)$, and it is easy to verify that the conditions of (ii) in Theorem 1 are satisfied. Figures 1-8 depict the equilibrium point $(0.075617,0.021169,0.44984,0.423379)$ which is locally asymptotically stable when $\tau=700<\tau_{0}$, while Figures 9-16 display the equilibrium point $(0.075617,0.021169$, $0.44984,0.423379$ ) that is unstable, and Hopf bifurcation occurs when $\tau=\tau_{0}$ (see Supplementary Materials (available here)). 


\section{Conclusion}

The problem of bifurcation for a fractional land model with time delay has been studied in this paper. Firstly, by comprehensively analyzing the characteristic equation, the stability criterion and the conditions of existence of Hopf bifurcation are established. It was shown that the presence of delay heavily affects the stability of the model. Finally, according to China, Yunan Province, Yuxi city land data, some simulation examples have been exploited to illustrate the applicability and usefulness of the developed theoretical result.

\section{Data Availability}

The labeled dataset used to support the findings of this study is available from the corresponding author upon request.

\section{Conflicts of Interest}

The authors declare that there are no conflicts of interest regarding the publication of this paper.

\section{Acknowledgments}

This work was supported by the National Natural Science Foundation of China under Grant nos. 61903323 and 11361072, the Natural Scientific Research Fund Project of Yunnan Province under Grant nos. 2017FH001-045 and 2018FH001-012, and the National Key Research and Development Project under Grant no. 2016YFC0502406.

\section{Supplementary Materials}

In this paper, in "Section 4," according to Yuxi city land and population data of Yunan Province, China, that is to say, from numerical simulation of data (see attachment of data.txt, basic data), we have $a=0.34, b=0.012, r=0.0048$, $s=0.004, h=0.05$, and $d=0.08$. By computing, we obtain the positive equilibrium point as $(0.075617,0.021169,0.44984$, 0.423379). Moreover, we have $R_{0}=((b d+d s) / a s h)=$ $18.8235>1, \omega_{0}=0.002358$, and $\tau_{0}=728.403$. Furthermore, by using software of mathematics, we obtain fitting results, that is, it is in the attachments data1.txt and data2.txt (data after numerical simulation). As a matter of fact, this shows that numerical simulation is consistent with the actual, see Figures 1-8 and Figures 9-16. (Supplementary Materials)

\section{References}

[1] B. W. Huang, "On earth system science and sustainable development strategy (I)," Acta Geographica Sinica, vol. 51, pp. 350-354, 1996, in Chinese.

[2] W. V. Reid, D. Chen, L. Goldfarb et al., "Earth system science for global sustainability: grand challenges," Science, vol. 330, no. 6006, pp. 916-917, 2010.

[3] B. L. Turner II, "Local faces, global flows: the role of land use and land cover in global environmental change," Land Degradation and Development, vol. 5, no. 2, pp. 71-78, 1994.
[4] X. B. Li, "A review of the international researches on land use/ land cover change," Acta Geographica Sinica, vol. 51, pp. 553-557, 1996, in Chinese.

[5] A. M. Dewan and Y. Yamaguchi, "Land use and land cover change in greater Dhaka, Bangladesh: using remote sensing to promote sustainable urbanization," Applied Geography, vol. 29, no. 3, pp. 390-401, 2009.

[6] H. J. Geist and E. F. Lambin, "Proximate causes and underlying driving forces of tropical deforestation," Bioscience, vol. 52, no. 2, pp. 143-150, 2002.

[7] S. Rost, D. Gerten, A. Bondeau, W. Lucht, J. Rohwer, and S. Schaphoff, "Agricultural green and blue water consumption and its influence on the global water system," Water Resources Research, vol. 44, pp. 137-148, 2008.

[8] L. H. Hou and Y. L. Cai, "An essential analysis and review on land use/cover change research," Progress in Geography, vol. 23, pp. 96-103, 2004, In Chinese.

[9] E. O'Rourke, "Socio-natural interaction and landscape dynamics in the Burren, Ireland," Landscape and Urban Planning, vol. 70, no. 1-2, pp. 69-83, 2005.

[10] J. Ni and J. A. Shao, "The drivers of land use change in the migration area, three gorges project, China: advances and prospects," Journal of Earth Science, vol. 24, no. 1, pp. 136-144, 2013.

[11] N. K. Msofe, L. X. Sheng, and J. Lyimo, "Land use change trends and their driving forces in the Kilombero valley floodplain, Southeastern Tanzania," Sustainability, vol. 11, no. 2, p. 505, 2019.

[12] J. Kleemann, G. Baysal, H. N. N. Bulley, and C. Frst, "Assessing driving forces of land use and land cover change by a mixed-method approach in north-eastern Ghana, West Africa," Journal of Environmental Management, vol. 196, pp. 411-442, 2017.

[13] D. Y. Liu, X. Q. Zheng, and H. B. Wang, "Land-use simulation and decision-support system (landSDS): seamlessly integrating system dynamics, agent-based model, and cellular automata," Ecological Modelling, vol. 417, pp. 1-10, 2020.

[14] Y. Q. Chen, H. V. Peter, and B. Xu, "Spatial modeling of land use and its effects in China," Progress in Geography, vol. 19, pp. 116-127, 2000, in Chinese.

[15] J. Zhang, W. B. Zhu, S. Y. Wu, and S. C. Li, "Simulation of temporal and special land use changes in Jing-Jin-Ji urban agglomeration using CLUE-S model," Acta Scientiarum Naturalium Universitatis Pekinensis, vol. 54, pp. 115-124, 2018, in Chinese.

[16] J. J. Liu, M. Xia, Y. Z. Liu, K. L. Zhang, and Z. H. Zhang, "Driving mechanism of rural land use change based on multiagent system and cellular automata," Transactions of the Chinese Society of Agricultural Engineering, vol. 34, pp. 242252, 2018, in Chinese.

[17] A. P. Dobson, A. D. Bradshaw, and A. J. M. Baker, "Hopes for the future: restoration ecology and conservation biology," Science, vol. 277, no. 5325, pp. 515-522, 1997.

[18] S. S. Chen, J. Hua, F. X. Zhang, and Y. M. Li, "Dynamical analysis of land model with Holling II land reclamation rate," Journal of Systems Science and Complexity, vol. 37, pp. 819827, 2017.

[19] I. Kubiaczyk and S. H. Saker, "Oscillation and stability in nonlinear delay differential equations of population dynamics," Mathematical and Computer Modelling, vol. 35, no. 3-4, p. 295, 2002.

[20] K. Gopalsamy and I. Leung, "Delay induced periodicity in a neural netlet of excitation and inhibition," Physica D, vol. 89, no. 3-4, pp. 395-426, 1996. 
[21] Y. H. Xia and V. G. Romanovski, "Bifurcation analysis of a population dynamics in a critical state," Bulletin of the Malaysian Mathematical Sciences Society, vol. 38, no. 2, pp. 499-527, 2015.

[22] Z. Wei, Y. H. Xia, and T. H. Zhang, "Stability and bifurcation analysis of a amensalism model with weak Allee effect," Qualitative Theory of Dynamical Systems, vol. 19, no. 1, pp. 19-23, 2020.

[23] X. D. Li, J. H. Shen, and R. Rakkiyappan, "Persistent impulsive effects on stability of functional differential equations with finite or infinite delay," Applied Mathematics and Computation, vol. 329, pp. 14-22, 2018.

[24] X. Y. Yang, X. D. Li, X. Li, Q. Xi, and P. Y. Duan, "Review of stability and stabilization for impulsive delayed systems," Mathematical Biosciences \& Engineering, vol. 15, no. 6, pp. 1495-1515, 2018.

[25] X. D. Li, X. Y. Yang, and T. W. Huang, "Persistence of delayed cooperative models: impulsive control method," Applied Mathematics and Computation, vol. 342, pp. 130-146, 2019.

[26] D. Yang, X. D. Li, J. Shen, and Z. J. Zhou, "State-dependent switching control of delayed switched systems with stable and unstable modes," Mathematical Methods in the Applied Sciences, vol. 41, no. 16, pp. 6968-6983, 2018.

[27] D. Yang, X. D. Li, and J. L. Qiu, "Output tracking control of delayed switched systems via state-dependent switching and dynamic output feedback," Nonlinear Analysis: Hybrid Systems, vol. 32, pp. 294-305, 2019.

[28] Y. Q. Wang, J. Q. Lu, and Y. J. Lou, "Halanay-type inequality with delayed impulses and its applications," Science China: Information Sciences, vol. 62, no. 9, Article ID 192206, 2019.

[29] E. Ahmed, A. M. A. El-Sayed, and H. A. A. El-Saka, "Equilibrium points, stability and numerical solutions of fractionalorder predator-prey and rabies models," Journal of Mathematical Analysis and Applications, vol. 325, no. 1, pp. 542-553, 2007.

[30] C. D. Huang, J. D. Cao, M. Xiao, A. Alsaedi, and F. E. Alsaadi, "Controlling bifurcation in a delayed fractional predator-prey system with incommensurate orders," Applied Mathematics and Computation, vol. 293, pp. 293-310, 2017.

[31] S. Das, P. K. Gupta, and Rajeev, "A fractional predator-prey model and its solution," International Journal of Nonlinear Sciences and Numerical Simulation, vol. 10, no. 7, pp. 873-876, 2009.

[32] H. A. El-Saka, E. Ahmed, M. I. Shehata, and A. M. A. El-Sayed, "On stability, persistence, and Hopf bifurcation in fractional order dynamical systems," Nonlinear Dynamics, vol. 56, no. 12, pp. 121-126, 2009.

[33] C. Luo and X. Y. Wang, "Chaos generated from the fractionalorder complex chen system and its application to digital secure communication," International Journal of Modern Physics C, vol. 24, no. 04, p. 1350025, 2013.

[34] E. Kaslik and S. Sivasundaram, "Nonlinear dynamics and chaos in fractional-order neural networks," Neural Networks, vol. 32, no. 1, pp. 245-256, 2012.

[35] Y. Li, H. P. Wang, and Y. Tian, "Fractional-order adaptive controller for chaotic synchronization and application to a dual-channel secure communication system," Modern Physics Letters B, vol. 33, no. 24, pp. 1097-1106, 2019.

[36] L. X. Yang, W. S. He, and X. J. Liu, "Synchronization between a fractional-order system and an integer order system," Computers \& Mathematics with Applications, vol. 62, no. 12, pp. 4708-4716, 2011.

[37] M. Odai and Y. Hori, "Controller design robust to nonlinear elements based on fractional order control system," IEEE
Transactions on Industry Applications, vol. 120, no. 1, pp. 11-18, 2010.

[38] C. D. Huang, H. Liu, X. P. Chen, M. S. Zhang, J. D. Cao, and A. Alsaedi, "Dynamic optimal control of enhancing feedback treatment for a delayed fractional order predator-prey model," Physica A: Statistical Mechanics and Its Applications, vol. 554, Article ID 124136, 2020.

[39] Z. Wang, Y. K. Xie, J. W. Lu, and Y. X. Li, "Stability and bifurcation of a delayed generalized fractional-order preypredator model with interspecific competition," Applied Mathematics and Computation, vol. 347, pp. 360-369, 2019.

[40] C. D. Huang, Z. H. Li, D. W. Ding, and J. D. Cao, "Bifurcation analysis in a delayed fractional neural network involving selfconnection," Neurocomputing, vol. 314, pp. 186-197, 2018.

[41] D. Matignon, "Stability results for fractional differential equations with applications to control processing," in Proceedings of the Computational Engineering in Systems and Application Multi-Conference, IMACS, vol. 2, pp. 963-968, Lille, France, 2016.

[42] X. Li and R. C. Wu, "Hopf bifurcation analysis of a new commensurate fractional-order hyperchaotic system," Nonlinear Dynamics, vol. 78, no. 1, pp. 279-288, 2014.

[43] N. Laskin, "Fractional quantum mechanics," Physical Review E, vol. 62, no. 3, pp. 3135-3145, 2000.

[44] I. Podlubny, Fractional Differential Equations, Academic Press, Cambridge, MA, USA, 1999.

[45] A. A. Kilbas, H. M. Srivastava, and J. J. Trujillo, Theory and Application of Fractional Differential Equations, Elsevier, New York, NY, USA, 2006.

[46] T. Zhao, Z. Z. Zhang, and R. K. Upadhyay, "Delay-induced Hopf bifurcation of an SVEIR computer virus model with nonlinear incidence rate," Advances in Difference Equations, vol. 2018, no. 1, 2018.

[47] E. Hanert, E. Schumacher, and E. Deleersnijder, "Front dynamics in fractional-order epidemic models," Journal of Theoretical Biology, vol. 279, no. 1, pp. 9-16, 2011.

[48] C. Vargas-De-León, "Volterra-type Lyapunov functions for fractional-order epidemic systems," Communications in Nonlinear Science and Numerical Simulation, vol. 24, no. 1-3, pp. 75-85, 2015.

[49] H. AbdelhamedAsfour and M. Ibrahim, "On the differential fractional transformation method of MSEIR epidemic model," International Journal of Computer Applications, vol. 113, no. 3, pp. 10-16, 2015.

[50] W. Zhu, Y. H. Xia, B. Zhang, and Y. Bai, "Exact traveling wave solutions and bifurcations of the time fractional differential equations with applications," International Journal of Bifurcation and Chaos, vol. 29, no. 3, Article ID 1950041, 2019.

[51] A. S. Deshpande, V. Daftardar-Gejji, and Y. V. Sukale, "On Hopf bifurcation in fractional dynamical systems," Chaos, Solitons \& Fractals, vol. 98, pp. 189-198, 2017.

[52] H. Wang, Y. Yu, G. Wen, and S. Zhang, "Stability analysis of fractional-order neural networks with time delay," Neural Processing Letters, vol. 42, no. 2, pp. 479-500, 2015.

[53] M. Xiao, W. X. Zheng, G. Jiang, and J. D. Cao, "Undamped oscillations generated by Hopf bifurcations in fractional-order recurrent neural networks with caputo derivative," IEEE Transactions on Neural Networks and Learning Systems, vol. 26, no. 12, pp. 3201-3214, 2015.

[54] S. Bhalekar and D. Varsha, "A predictor-corrector scheme for solving nonlinear delay differential equations of fractional order," Journal of Fractional Calculus and Applications, vol. 1, pp. 1-9, 2011. 